\title{
Reply to Ward and Colleagues' Comment on “Using Human Experiments of Nature to Predict Drug Safety Issues: An Example with PCSK9 Inhibitors"
}

\author{
Rebecca N. Jerome ${ }^{1}$. Jill M. Pulley ${ }^{1}$ - Dan M. Roden ${ }^{2}$. Jana K. Shirey-Rice ${ }^{1} \cdot$ Lisa A. Bastarache $^{3}$. Gordon Bernard ${ }^{1,2}$. \\ Leeland Ekstrom ${ }^{1,4} \cdot$ William J. Lancaster ${ }^{3} \cdot$ Joshua C. Denny $^{3}$
}

Published online: 31 July 2018

(c) Springer Nature Switzerland AG 2018

We appreciate Ward and colleagues' interest in our work [1] and the opportunity to respond. We confirm that the spina bifida cases we reviewed are carriers of the R46L variant minor allele. As acknowledged in our paper, both the size of our observed spectrum of $p$ values and the limited spectrum of PCSK9 variants in our existing genotyping platform suggest our data may be underpowered for fully understanding the connections identified. We note that the most conservative Bonferroni correction for multiplicity in this dataset would be approximately $6 \times 10^{-6}$, and the authors are correct that our association with neural tube defects is below this threshold. The association with a diagnosis of hypercholesterolemia, a known association with the R46L variant, is also below the threshold. However, the existence of animal and human data suggestive of a potential link between PCSK9 and spina bifida, in concordance with the association we observed, suggests prudence in safety signal reporting is warranted. As described in our paper, decreased PCSK9 was found in both a rat fetal model of neural tube defects and women carrying a fetus affected by a neural tube defect, with PCSK9 level additionally noted to be a potentially useful biomarker in humans, with sensitivity of 57\% and specificity of $98 \%$ [2]. Furthermore, the UK Biobank is

This reply refers to the article available at https://doi.org/10.1007/ s40264-018-0702-y.

\section{Rebecca N. Jerome}

rebecca.jerome@vumc.org

1 Vanderbilt Institute for Clinical and Translational Research, Vanderbilt University Medical Center, Nashville, TN, USA

2 Office of Research, Vanderbilt University Medical Center, Nashville, TN, USA

3 Department of Biomedical Informatics, Vanderbilt University School of Medicine, Nashville, TN, USA

4 Nashville Biosciences, Nashville, TN, USA an epidemiologic cohort of middle-aged subjects and thus may underrepresent phenotypes such as spina bifida seen primarily in younger patients.

\section{Compliance with Ethical Standards}

Funding The project described herein is supported by Clinical and Translational Science Award number UL1 TR002243 from the National Center for Advancing Translational Sciences. Its contents are solely the responsibility of the authors and do not necessarily represent the official views of the National Center for Advancing Translational Sciences or the National Institutes of Health.

Conflict of interest Rebecca N. Jerome, Jill M. Pulley, Dan M. Roden, Jana K. Shirey-Rice, Lisa A. Bastarache, Gordon Bernard, Leeland Ekstrom, William J. Lancaster, and Joshua C. Denny have no conflicts of interest to declare that are directly relevant to the content of this reply.

\section{References}

1. Ward LD, Moffat GJ, Yuan J, Nioi P. Comment on "Using Human Experiments of Nature to Predict Drug Safety Issues: An Example With PCSK9 Inhibitors". Drug Saf. 2018. https://doi.org/10.1007/ s40264-018-0702-y.

2. An D, Wei X, Li H, Gu H, Huang T, Zhao G, et al. Identification of PCSK9 as a novel serum biomarker for the prenatal diagnosis of neural tube defects using iTRAQ quantitative proteomics. Sci Rep. 2015;5:17559. 